\title{
An ILI Mitigating Modulation Scheme for Molecular MIMO Communications
}

\author{
Mustafa Can Gursoy*, Ertugrul Basar ${ }^{\dagger}$, Ali Emre Pusane*, Tuna Tugcu ${ }^{\ddagger}$ \\ *Department of Electrical and Electronics Engineering, Bogazici University, Istanbul, Turkey \\ ${ }^{\dagger}$ Department of Electrical and Electronics Engineering, CoreLab, Koç University, Istanbul, Turkey \\ ${ }^{\ddagger}$ Department of Computer Engineering, NETLAB, Bogazici University, Istanbul, Turkey \\ E-mail: \{can.gursoy, ali.pusane, tugcu\}@boun.edu.tr, ebasar@ku.edu.tr
}

\begin{abstract}
Multiple-input-multiple-output (MIMO) approach is recently proposed to the diffusion-based molecular communication realm in order to enhance communication performance. One of the most promising approaches of molecular MIMO, spatial multiplexing (SMUX), transmits parallel streams of data to achieve an increased throughput. Unfortunately, the performance of SMUX is limited by a high error floor due to severe interlink interference (ILI). Inspired by the relatively more sparse transmission strategy of the pulse-position modulation (PPM) scheme, this study proposes a PPM-based novel SMUX approach to the molecular MIMO literature. In the study, it is shown that the proposed approach combats ILI more effectively than the existing concentration-based SMUX approach and yields promising error performances.
\end{abstract}

Keywords-Diffusion-based molecular communications, MIMO, nanonetworks, pulse-position modulation, spatial multiplexing.

\section{INTRODUCTION}

Diffusion-based molecular communications (DBMC) is a paradigm that utilizes the free diffusion of molecules to convey information [1]. In a DBMC system, the transmitted message can be encoded in the quantity [2], type [3], time of release [4], and possibly other properties of the emitted molecules. These molecules are called messenger molecules (MMs) and they exhibit Brownian motion after their release into the fluid communication environment.

The Brownian motion-induced random propagation of MMs causes some molecules to take longer times to arrive at the receiver. In a time slotted DBMC system, these molecules may arrive at the receiver in later time slots than the intended one and cause inter-symbol interference (ISI) [5]. ISI is an important bottleneck of a DBMC system and is known to deteriorate the error performance for higher data rates.

In order to achieve higher data rates while maintaining a sufficiently low error performance, the multiple-input-multipleoutput (MIMO) setting is proposed to the DBMC domain. One of such works utilizes a $2 \times 2$ molecular MIMO setting to implement an Alamouti space-time block code and a repetition coding scheme [6]. A promising approach to utilize the available antennas is to transmit parallel data streams from each transmitter and receiver antenna pair [7]-[9] for each

This work was supported by State Planning Organization of Turkey (DPT) under grant number DPT-2007K120610. channel use, which can be considered analogous to a spatial multiplexing (SMUX) approach.

SMUX is able to transmit multiple symbols for each channel use, which helps the system in terms of ISI combating while satisfying the specified bit rate constraint. However, since MMs that correspond to independent symbols are emitted for each transmitter and receiver antenna link, the random propagation of MMs cause significant inter-link interference (ILI) and imposes an error floor.

Inspired by the promising results of [10] regarding the use of higher order pulse-position modulation (PPM) schemes in DBMC, the main contribution of this study is the utilization of PPM symbols in conjunction with the SMUX approach in order to mitigate ILI. The study emphasizes that the relatively more sparse transmission strategy of PPM is more suitable to SMUX than using concentration shift keying (CSK) symbols in terms of ILI combating. Furthermore, the simplicity of the PPM detector is also an advantage over CSK's threshold-based detector since PPM's decoder does not require the channel impulse response (CIR). The findings of this work also agree with [11] that the ILI associated with SMUX increases for larger symbol durations. Stemming from this phenomenon, this work suggests the existence of a trade-off between ISI and ILI combating and hints to the presence of a bit duration value in which SMUX schemes yield their lowest error probabilities.

\section{SYSTEM MODEL}

Following the system model considerations of [7], [9] and [11], the system in this paper is considered to be an $N \times N$ molecular MIMO system, where $N=4$ is chosen for demonstrative purposes. In the molecular MIMO system of interest, there is a single transmitter unit and a single receiver unit. The transmitter and receiver are assumed to be equipped with four emission points and four fully absorbing spherical receivers that act as transmit and receiver antennas, respectively. The corresponding antennas are assumed to be aligned against each other. The transmitter unit's body is considered to be fully permeable to the MMs whilst the receiver unit is considered to be perfectly reflective. Other than the transmitter and receiver bodies, the considered communication environment is unbounded, three dimensional, and driftless, which allows the MMs to exhibit Brownian motion with a diffusion coefficient of $D$ [12]. When communicating, the transmitter and receiver 


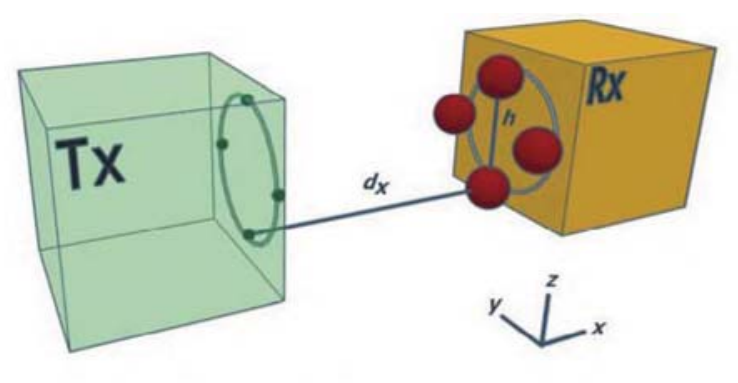

Fig. 1. The $4 \times 4$ molecular MIMO system model considered in the paper.

units are assumed to be accurately synchronized with each other using a method similar to as mentioned in [13]. Overall, the system model of interest is presented in Fig. 1.

In the system model presented in Fig. $1, d_{x}$ denotes the closest distance between a transmit and receiver antenna pair. Furthermore, it is observable from Fig. 1 that the antennas are placed on a uniform circular array (UCA) in this demonstrative model with $\frac{\pi}{2}$ radians of angular difference between them. The distance between the opposite receivers of the UCA is denoted by $h$, as also shown in Fig. 1. Given that the radius of a single spherical receiver antenna is denoted by $r_{r}$, the radius of the transmitter UCA is $\frac{h}{2}+r_{r}$.

\section{PROPOSED APPROACH}

\section{A. Channel Model \& The Existing SMUX Approach}

Recall that the molecular MIMO setting considered in this paper consists of four transmit and four receiver antennas that are aligned against to each other, forming four pairs. The SMUX approach presented in [7] and [9] utilizes all these pairs to transmit independent bit streams. In a time slotted $N \times N$ molecular MIMO system, these independent transmissions correspond to dividing the message sequence into groups of $N$ bits/symbols, mapping each bit/symbol according to the utilized modulation scheme, and emitting the MMs. The existing SMUX approach ([7]) utilizes binary CSK (BCSK) symbols with on-off keying as the modulation scheme.

In the time slotted diffusive molecular MIMO channel model, the number of arriving molecules at a receiver antenna is found by superposing the effects of all transmit antennas on it [6]-[8]. To characterize the channel, we start by denoting $p_{i, j}[k]$ as the $k^{t h}$ channel coefficient corresponding to the subchannel between the $i^{\text {th }}$ transmitter and $j^{\text {th }}$ receiver antenna. $p_{i, j}[k]$ represents the probability of a single molecule that was emitted from the $i^{\text {th }}$ transmit antenna arriving at the $j^{\text {th }}$ receiver antenna at the $k^{\text {th }}$ time slot. Due to the presence absorbing receivers, $p_{i, j}[k]$ need to be found by random walkbased Monte Carlo simulations or with the help of artificial neural networks [14]. In this paper, random walk-based Monte Carlo simulations are utilized to generate $p_{i, j}[k]$.

When considering multiple molecule transmissions, we utilize the the independent Gaussian approximation employed in [6]-[8] and [15]. Therefore, the arrival at the $j^{\text {th }}$ receiver antenna at the $k^{t h}$ time slot, $R_{j}[k]$, can be approximately represented as $R_{j}[k] \sim \mathcal{N}\left(\mu_{j}[k], \sigma_{j}^{2}[k]\right)$, where the mean is found by

$$
\mu_{j}[k]=\sum_{z=k-L+1}^{k} \sum_{i=1}^{N} s_{i}[z] p_{i, j}[k-z+1]
$$

and the variance by

$$
\sigma_{j}^{2}[k]=\sum_{z=k-L+1}^{k} \sum_{i=1}^{N} s_{i}[z] p_{i, j}[k-z+1]\left(1-p_{i, j}[k-z+1]\right) .
$$

Here, $s_{i}[z]$ denotes the number of molecules transmitted from the $i^{t h}$ transmit antenna at time index $z$ and $L$ denotes the channel memory (including the intended tap). In this paper, $L=30$ is chosen for BCSK-based SMUX in order to fully capture the heavy right of the arrival distribution.

\section{B. Proposed SMUX Scheme}

Overall, this study considers utilizing PPM constellations to transmit information in a spatial multiplexing-based molecular MIMO system. In the proposed scheme, the original bit stream is divided into $N$ parallel streams called $\mathbf{u}_{i}$ where $i=1, \ldots, N$, which denote the assigned bit sequence for the $i^{t h}$ transmit and receiver antenna pair. Each $\mathbf{u}_{i}$ is then further divided into $\log _{2} \beta$-bit long sequences with the $m^{\text {th }}$ sequence denoted as $\mathbf{x}_{i}[m](i=1, \ldots, N)$, where $\beta$ is the order of the utilized PPM scheme. Lastly, each $\mathbf{x}_{i}[m]$ is mapped to its corresponding PPM chip sequence according to the mapping mentioned in [10]. Throughout the paper, each PPM sub-slot is referred to as a chip. The overall scheme is aptly called $\beta$ PPM SMUX in the paper. The transmission strategy for 8-PPM SMUX is presented in Table I for demonstrative purposes.

TABLE I

TRANSMISSION STRATEGY FOR EACH TRANSMITTER - RECEIVER ANTENNA LINK WHEN USING 8-PPM SMUX

\begin{tabular}{|c|c|c|}
\hline Decimal & $\mathbf{x}_{i}[m]$ & PPM Chip Sequence \\
\hline 1 & 000 & 00000001 \\
\hline 2 & 001 & 00000010 \\
\hline 3 & 010 & 00000100 \\
\hline 4 & 011 & 00001000 \\
\hline 5 & 100 & 00010000 \\
\hline 6 & 101 & 00100000 \\
\hline 7 & 110 & 01000000 \\
\hline 8 & 111 & 10000000 \\
\hline
\end{tabular}

At the receiver end, the decoder stores the arrival counts for the $\beta$-chip long sequence when employing $\beta$-PPM SMUX and obtains $\hat{\mathbf{x}}_{i}[\mathrm{~m}]$ by performing an arg max operation on the stored chips. Note that this decoding operation does not require CIR unlike CSK-based methods, which is a desired property in terms of receiver complexity [10].

In order to obtain a fair comparison among different modulation schemes for molecular communications, there are two main normalizations that need to be imposed on them. Firstly, considering that the data rate directly affects the ISI a system faces, the compared schemes need to be evaluated for the same bit rate. Therefore, bit duration is the first main fairness constraint and is denoted by $t_{b}$ in this paper. Second, the 
number of emitted molecules also need to be normalized on a per-bit basis, since it directly relates to the energy consumed by a modulation scheme to transmit a single bit [16]. The number of emitted molecules per bit constraint is denoted by $M$ in this work. Note that these per-bit constraints imply that the more bits a modulation scheme can transmit per channel use, the more molecules it can use for each symbol transmission.

Since it can transmit $N$ parallel streams of 1-bit long symbols simultaneously, BCSK-based SMUX can transmit at a symbol duration of $N t_{b}$, while still satisfying the bit duration constraint of $t_{b}$. Note that transmitting at $N t_{b}$ duration allows BCSK-based SMUX to combat ISI very effectively without sacrificing from throughput. Similarly, 2-PPM SMUX can also transmit at $N t_{b}$ due to a single 2-PPM symbol also having one bit of information embedded in it. This corresponds to each chip of 2-PPM SMUX to have a chip duration of $\frac{N t_{b}}{2}$. On the other hand, 4-PPM SMUX is able to encode 2-bit long symbols in four chips, which allows its chips to be sent at $\frac{2 N t_{b}}{4}$ duration. Similarly, the chip duration of $\frac{3 N t_{b}}{8}$ can be obtained for 8-PPM SMUX.

Recall that the number of emitted molecules per each transmitted bit is represented by $M$. This naturally allows each symbol in 2-PPM SMUX to be transmitted using $M$ molecules. Similarly, 4-PPM SMUX and 8-PPM SMUX are allowed to transmit using $2 M$ and $3 M$ molecules per symbol per antenna. In this paper, the transmitted bits are assumed to be independent, and the probability of transmitting a bit1 or bit- 0 is considered equal, with both options having $P_{1}=P_{0}=0.5$ probability. Therefore, the on-off keying-based BCSK SMUX transmits a bit- 1 with $2 M$ molecules and bit- 0 with 0 molecules in order to satisfy the $M$ constraint.

\section{NUMERICAL RESULTS AND DISCUSSION}

In this section, comparative bit error rate (BER) performances of BCSK-based SMUX as considered by [7] and the proposed PPM-based SMUX are presented. One thing to note is that in the performed computer simulations, the channel memory (the value of $L$ ) is normalized according to the slot durations of the PPM schemes. For example, since the slot durations of 2 and 4-PPM are both equal to $\frac{N t_{b}}{2}$, they are subjected to a 60-tap channel rather than BCSK-based SMUX's 30-tap channel. Firstly, Fig. 2 is presented to provide a comparison at different bit rates.

The results of Fig. 2 show that PPM-based SMUX schemes outperform CSK-based SMUX for the majority of $t_{b}$ values and yield promising error performances. The main reason for PPM-based schemes' better performance is the fact that they combat ILI more effectively than CSK. When using CSK for each transmitter and receiver antenna pair, the system utilizes every slot interval to transmit information ${ }^{1}$, which results in emitting $\frac{N}{2}$ simultaneous molecular pulses to the MIMO channel on average, given $P_{1}=P_{0}=0.5$. This transmission

\footnotetext{
${ }^{1}$ Note that each slot corresponds to an individual bit interval for a CSK scheme.
}

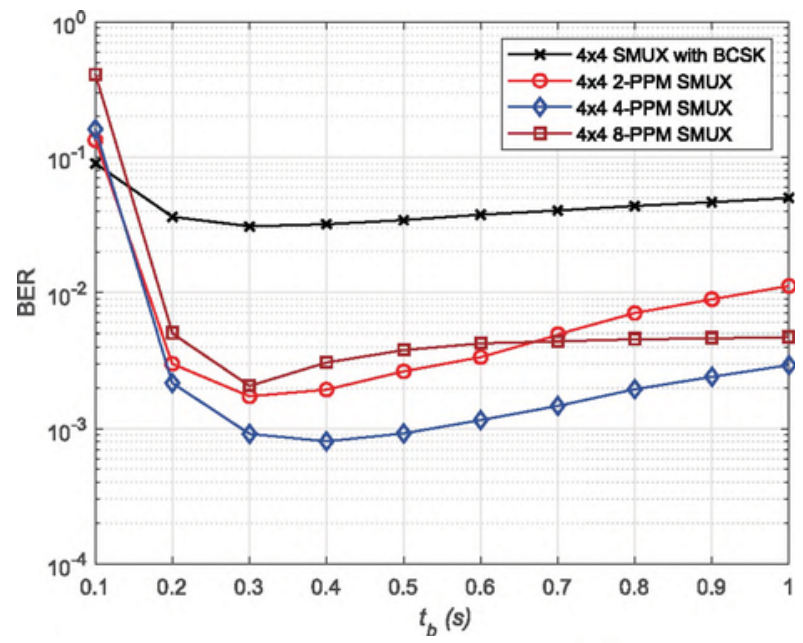

Fig. 2. BER vs. $t_{b}$ curves for BCSK and PPM-based SMUX schemes. $M=$ 750 molecules, $d_{x}=8 \mu \mathrm{m}, h=6 \mu \mathrm{m}, D=79.4 \frac{\mu \mathrm{m}^{2}}{\mathrm{~s}}, r_{r}=5 \mu \mathrm{m}$.

strategy introduces severe ILI due to MMs propagating randomly and being able to arrive at unintended receivers, which imposes an irreducable error floor [17]. This effect is mitigated when using PPM schemes as constellations, since PPM's very nature reduces the probability of simultaneous transmissions.

As can also be observed from Fig. 2, one exception to PPM's overall better error performance is at $t_{b}=0.1 \mathrm{~s}$. With decreasing $t_{b}$, the probability of the emitted MMs to arrive at the receiver at the intended time slot decreases, whilst the probability of arrivals for the interference slots increases. This adverse relation between the coefficients causes the small $t_{b}$ region to correspond to a high ISI region, as well. Note that for very a small value of $t_{b}=0.1 \mathrm{~s}$, the channel is subject to severe ISI. For extremely high ISI scenarios, PPM is known to suffer more than CSK-based schemes since it further divides the already short symbol duration into even shorter slot durations [10], which explains the inferior BER performance of PPMbased SMUX at $t_{b}=0.1 \mathrm{~s}$. As $t_{b}$ starts to increase, this effect is mitigated and PPM-based SMUX shows better error performances by being able to reach lower target BER values due to its efficient ILI-combating.

A noteworthy implication of Fig. 2 is that the BER of the SMUX schemes do not monotonically decrease as $t_{b}$ increases, even though ISI decreases with increasing $t_{b}$. This phenomenon is due to $\mathrm{ILI}$ increasing with $t_{b}$. Note that as the bit duration is increased, the MMs that take longer paths before their arrivals are more likely to reach one of the receiver antennas. Furthermore, compared to the MMs that take shorter times until their arrivals, these MMs have higher probabilities to arrive at a receiver antenna other than their intended ones. This phenomenon causes the arrival to become more uniformlike among receiver antennas, therefore increasing ILI. Since SMUX can combat ISI very effectively due to the discussion made in Subsection III-B, combating ISI by further increasing $t_{b}$ does not significantly contribute to reducing BER. On the 


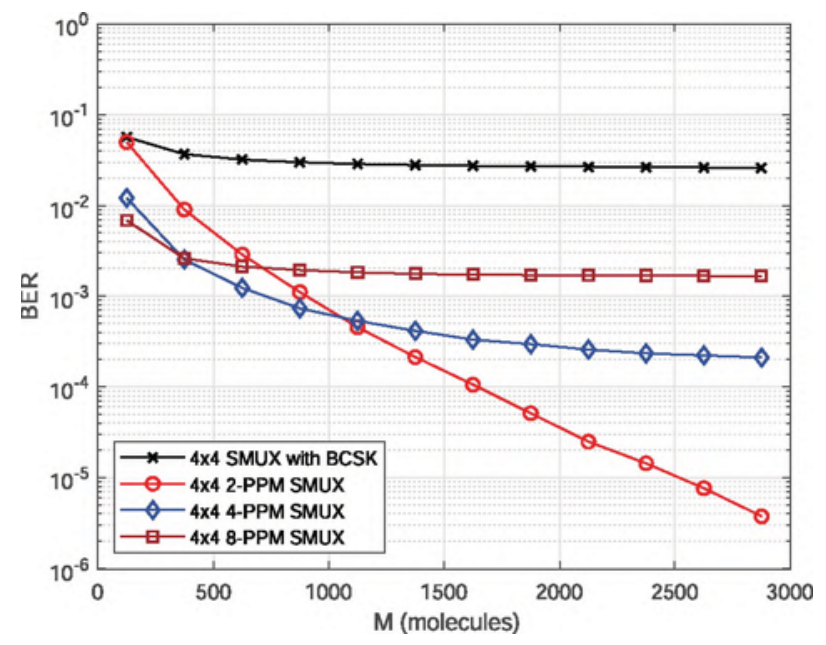

Fig. 3. BER vs. $M$ curves for BCSK and PPM-based SMUX schemes. $t_{b}=0.3 \mathrm{~s}, d_{x}=8 \mu \mathrm{m}, h=6 \mu \mathrm{m}, D=79.4 \frac{\mu \mathrm{m}^{2}}{\mathrm{~s}}, r_{r}=5 \mu \mathrm{m}$.

other hand, the introduced ILI enhancement hurts the SMUXbased schemes' error performances as $t_{b}$ is increased after a certain point. This phenomenon is also in agreement with the findings of [11] and implies an interesting possible research direction regarding the optimization of $t_{b}$ for SMUX-based molecular MIMO with an objective of minimizing BER.

For the molecular MIMO system described by the parameters in the description of Fig. 2, the results of Fig. 2 suggest that selecting $t_{b} \approx 0.3 \mathrm{~s}$ is beneficial for an objective of minimizing BER. Motivated by this parameter, Fig. 3 presents the BER vs. $M$ curves of the proposed schemes and BCSKbased SMUX evaluated at $t_{b}=0.3 \mathrm{~s}$. The results of Fig. 3 show that although at different BER values, SMUX schemes face error floors. This phenomenon can be simply explained by the fact that the MIMO DBMC channels are interference-limited channels and they are subject to both ISI and ILI. Among these two sources of interference, the limiting type of interference for SMUX is ILI. As also discussed in Paragraph 2 of this section, the ILI problem of BCSK-based SMUX mainly occurs due to simultaneous transmission of independent streams and can be alleviated by PPM-based SMUX since it inherently introduces possible time delays between emission pulses. Therefore, the fact that the possible time delay associated with 8-PPM SMUX being smaller can account for its inferior performance when compared to 2 and 4-PPM SMUX. Between 2 and 4-PPM SMUX, Fig. 3 shows that 4-PPM SMUX yields better error performance for lower $M$, whilst 2-PPM SMUX outperforms 4-PPM SMUX for higher $M$.

\section{ConClusion}

In this study, utilizing PPM symbols for SMUX-based molecular MIMO systems has been proposed. It has been shown through computer simulations that PPM-based SMUX outperforms the traditional CSK-based SMUX by being able to reach reliable target BERs and yields promising results. Furthermore, the presence of a local minimum of the BER vs. bit duration curve has been observed which hints to a trade-off between ISI and ILI combating of molecular MIMO schemes. Possible future work includes theoretical analysis of the proposed schemes' error performances, investigation of the performance loss for imperfect channel conditions (alignment issues, time-varying channels etc.), and derivation of a cost function of the BER vs. bit duration optimization problem.

\section{REFERENCES}

[1] N. Farsad, H. B. Yilmaz, A. Eckford, C. B. Chae, and W. Guo, "A comprehensive survey of recent advancements in molecular communication," IEEE Commun. Surveys Tuts., vol. 18, no. 3, pp. 1887-1919, Feb. 2016.

[2] M. S. Kuran, H. B. Yilmaz, T. Tugcu, and I. F. Akyildiz, "Modulation techniques for communication via diffusion in nanonetworks," in Proc. IEEE Intl. Conf. on Commun. (ICC), Apr. 2011.

[3] N.-R. Kim and C.-B. Chae, "Novel modulation techniques using isomers as messenger molecules for nano communication networks via diffusion," IEEE J. Sel. Areas Commun., vol. 31, no. 12, pp. 847-856, Dec. 2013.

[4] N. Garralda, I. Llatser, A. Cabellos-Aparicio, E. Alarcon, and M. Pierobon, "Diffusion-based physical channel identification in molecular nanonetworks," Nano Commun. Netw., vol. 2, no. 4, pp. 196-204, Dec. 2011.

[5] B. Tepekule, A. E. Pusane, H. B. Yilmaz, C.-B. Chae, and T. Tugcu, "ISI mitigation techniques in molecular communication," IEEE Trans. Mol. Biol. Multi-Scale Commun., vol. 1, no. 2, pp. 202-216, June 2015.

[6] M. Damrath, H. B. Yilmaz, C. Chae, and P. A. Hoeher, "Array gain analysis in molecular MIMO communications," IEEE Access, vol. 6 , pp. $61091-61102$, Oct. 2018.

[7] B. H. Koo, C. Lee, H. B. Yilmaz, N. Farsad, A. Eckford, and C.-B. Chae, "Molecular MIMO: From theory to prototype," IEEE J. Sel. Areas Commun., vol. 34, no. 3, pp. 600-614, Mar. 2016.

[8] B. H. Koo, H. B. Yilmaz, C. B. Chae, and A. Eckford, "Detection algorithms for molecular MIMO," in Proc. 2015 IEEE Intl. Conf. on Commun. (ICC), June 2015, pp. 1122-1127.

[9] L. S. Meng, P. C. Yeh, K. C. Chen, and I. F. Akyildiz, "MIMO communications based on molecular diffusion," in Proc. IEEE Global Commun. Conf. (GLOBECOM), Dec. 2012, pp. 5380-5385.

[10] B. C. Akdeniz, A. E. Pusane, and T. Tugcu, "Position-based modulation in molecular communications," Nano Commun. Netw., vol. 16, pp. 6068, June 2018.

[11] M. C. Gursoy, E. Basar, A. E. Pusane, and T. Tugcu, "Index modulation for molecular communication via diffusion systems," IEEE Trans. Commun., doi: 10.1109/TCOMM.2019.2898665.

[12] T. Nakano, A. W. Eckford, and T. Haraguchi, Molecular communication. Cambridge University Press, 2013.

[13] M. J. Moore and T. Nakano, "Oscillation and synchronization of molecular machines by the diffusion of inhibitory molecules," IEEE Trans. Nanotechnol., vol. 12, no. 4, pp. 601-608, July 2013.

[14] C. Lee, H. B. Yilmaz, C.-B. Chae, N. Farsad, and A. Goldsmith "Machine learning based channel modeling for molecular MIMO communications," in IEEE 18th Intl. Wkshp. on Sig. Proc. Adv. in Wireless Commun. (SPAWC), July 2017, pp. 1-5.

[15] H. B. Yilmaz, C.-B. Chae, B. Tepekule, and A. E. Pusane, "Arrival modeling and error analysis for molecular communication via diffusion with drift," in Proc. ACM 2nd Ann. Int. Conf. on Nanoscale Comput. and Commun., Sep. 2015.

[16] M. S. Kuran, H. B. Yilmaz, T. Tugcu, and B. Özerman, "Energy model for communication via diffusion in nanonetworks," Nano Commun. Netw., vol. 1, no. 2, pp. 86-95, July 2010.

[17] S. M. Rouzegar and U. Spagnolini, "Diffusive MIMO molecular communications: Channel estimation, equalization and detection," arXiv preprint arXiv:1809.06332, Sep. 2018. 